\title{
Reversibility of impaired brain structures after transsphenoidal surgery in Cushing's disease: a longitudinal study based on an artificial intelligence-assisted tool
}

\author{
*Bo Hou, MD, ${ }^{1}$ Lu Gao, MD, ${ }^{2-5}$ Lin Shi, PhD, ${ }^{6,7}$ Yishan Luo, PhD, 7 Xiaopeng Guo, MD, ${ }^{2,3}$ \\ Geoffrey S. Young, MD, ${ }^{4,5}$ Lei Qin, PhD, ${ }^{4,8}$ Huijuan Zhu, MD, ${ }^{\circ}$ Lin Lu, MD, ${ }^{9}$ Zihao Wang, MD, ${ }^{2,3}$ \\ Ming Feng, MD, , 2 Xinjie Bao, MD, ${ }^{2,3}$ Renzhi Wang, MD, ${ }^{2,3}$ Bing Xing, MD, ${ }^{2,3}$ and Feng Feng, MD ${ }^{1}$
}

Departments of ${ }^{1}$ Radiology and ${ }^{2}$ Neurosurgery, Peking Union Medical College Hospital, Chinese Academy of Medical Sciences and Peking Union Medical College, Beijing, China; ${ }^{3}$ China Pituitary Disease Registry Center, China Pituitary Adenoma Specialist Council, Beijing, China; ${ }^{4}$ Department of Radiology, Harvard Medical School, Boston, Massachusetts; ${ }^{5}$ Department of Radiology, Brigham and Women's Hospital, Boston, Massachusetts; ${ }^{\circ}$ Department of Imaging and Interventional Radiology, The Chinese University of Hong Kong, Shatin, Hong Kong, China; ${ }^{7 B}$ rainNow Research Institute, Shenzhen, Guangdong Province, China; ${ }^{8}$ Department of Imaging, Dana-Farber Cancer Institute, Boston, Massachusetts; and ${ }^{9}$ Key Laboratory of Endocrinology of the National Health Commission of the People's Republic of China, Department of Endocrinology, Peking Union Medical College Hospital, Chinese Academy of Medical Sciences and Peking Union Medical College, Beijing, China

\begin{abstract}
OBJECTIVE Cushing's disease (CD) involves brain impairments caused by excessive cortisol. Whether these impairments are reversible in remitted $C D$ after surgery has long been controversial due to a lack of high-quality longitudinal studies. In this study the authors aimed to assess the reversibility of whole-brain changes in remitted CD after transsphenoidal surgery (TSS), and its correlations with clinical and hormonal parameters, in the largest longitudinal study cohort to date for $\mathrm{CD}$ patient brain analysis.
\end{abstract}

METHODS Fifty patients with pathologically diagnosed $C D$ and 36 matched healthy controls (HCs) were enrolled in a tertiary comprehensive hospital and national pituitary disease registry center in China. 3-T MRI studies were analyzed using an artificial intelligence-assisted web-based autosegmentation tool to quantify 3D brain volumes. Clinical parameters as well as levels of serum cortisol, adrenocorticotrophic hormone (ACTH), and 24-hour urinary free cortisol were collected for the correlation analysis. All CD patients underwent TSS and 46 patients achieved remission. All clinical, hormonal, and MRI parameters were reevaluated at the 3-month follow-up after surgery.

RESULTS Widespread brain volume loss was observed in active CD patients compared with $\mathrm{HCs}$, including total gray matter ( $p=0.003$, with false discovery rate [FDR] correction) and the frontal, parietal, occipital, and temporal lobes; insula; cingulate lobe; and enlargement of lateral and third ventricles $(p<0.05$, corrected with FDR). All affected brain regions improved significantly after TSS ( $p<0.05$, corrected with FDR). In patients with remitted $C D$, total gray matter and most brain regions (except the frontal and temporal lobes) showed full recovery of volume, with volumes that did not differ from those of $\mathrm{HCs}(p>0.05$, corrected with FDR). ACTH and serum cortisol changes were negatively correlated with brain volume changes during recovery $(p<0.05)$.

CONCLUSIONS This study demonstrates the rapid reversal of total gray matter loss in remitted CD. The combination of full recovery areas and partial recovery areas after TSS is consistent with the incomplete recovery of memory and cognitive function observed in $\mathrm{CD}$ patients in clinical practice. Correlation analyses suggest that ACTH and serum cortisol levels are reliable serum biomarkers of brain recovery for clinical use after surgery.

https://thejns.org/doi/abs/10.3171/2019.10.JNS191400

KEYWORDS Cushing's disease; brain imaging; transsphenoidal surgery; artificial intelligence; pituitary surgery

ABBREVIATIONS ACTH = adrenocorticotrophic hormone; $C D$ = Cushing's disease; $C S$ = Cushing's syndrome; $D T I$ = diffusion tensor imaging; FDR = false discovery rate; $\mathrm{GC}=$ glucocorticoid; HC = healthy control; TSS = transsphenoidal surgery; $24 \mathrm{hUFC}=24$-hour urinary free cortisol.

SUBMITTED May 19, 2019. ACCEPTED October 25, 2019

INCLUDE WHEN CITING Published online January 3, 2020; DOI: 10.3171/2019.10.JNS191400.

${ }^{*}$ B.H. and L.G. share first authorship of this work. 
$\mathrm{C}$ USHING'S disease (CD), caused by adrenocorticotrophic hormone (ACTH)-secreting pituitary adenoma, is the most common cause of endogenous Cushing's syndrome (CS), which is characterized by elevated serum cortisol. ${ }^{19,22}$ In addition to the typical physical manifestations of central obesity and rounded face, CD causes cognitive impairment and memory deficits thought to be related to changes in brain structures rich in glucocorticoid (GC) receptors. ${ }^{9,23,34}$

Transsphenoidal surgery (TSS), the first-line treatment for $\mathrm{CD}$, yields remission rates of $60 \%-90 \%$. 40 Although impaired memory and cognitive functions generally improve significantly after surgery, many patients do not fully recover. ${ }^{28,38}$ Whether the effects of elevated cortisol on the brain in CD patients are "reversible" remains controversial even after meta-analysis. ${ }^{3}$ There are multiple reasons for this controversy, including the limited number of studies and their rather small sample sizes, ${ }^{3}$ predominant use of cross-sectional study designs, ${ }^{3}$ inclusion of study patients with heterogeneous origins of CS, ${ }^{28}$ and use of low-field MRI (1.0 T or $1.5 \mathrm{~T}$ ) with relatively low resolution. ${ }^{5,12,15,21,35-37,39}$ Furthermore, most previous studies did not exclude patients treated with radiotherapy prior to surgery, raising the possibility that observed brain changes could have been caused by radiotherapy. ${ }^{32}$ In addition, the 8 studies that reported long-term reversibility of the negative effects of elevated cortisol over 1 year of follow-up did not assess short-term brain changes..$^{5,12,15,21,35-37,39}$

To address these limitations in previously reported studies, we enrolled $50 \mathrm{CD}$ patients who had no previous medical or radiological therapy and 36 healthy controls (HCs), thereby creating the largest longitudinal cohort to date for the study of brain structural changes associated with CD. With the help of high-resolution 3-T MRI and an artificial intelligence-assisted web-based autosegmentation and quantification tool, we were able to quantify the 3D structural changes in the brain with high anatomical validity. To the best of our knowledge, this study is the first longitudinal study to evaluate whole-brain changes in CD patients during short-term remission. To better understand the correlation between clinical parameters and the reversibility of brain structure changes, we also correlated these brain changes with age, sex, duration of disease, years of education, and levels of serum cortisol, adrenocorticotrophic hormone (ACTH), and 24-hour urinary free cortisol (24hUFC).

\section{Methods}

\section{Subjects}

Fifty right-handed CD patients undergoing surgery at the Department of Neurosurgery, Peking Union Medical College Hospital, between January 2015 and April 2016 were enrolled. Thirty-six right-handed HCs were recruited who had no history of GC treatment and were matched with the study patients for age, sex, and years of education. The exclusion criteria included history of previous radiotherapy, drug or alcohol abuse, brain trauma, present or past neurological diseases, and contraindications to MRI. The diagnosis of CD was achieved by a combination of dynamic gadolinium-enhanced MRI of the pi- tuitary gland, low-dose dexamethasone suppression test, high-dose dexamethasone suppression test, inferior petrosal sinus sampling, and postsurgical pathology. Detailed preoperative assessments of diagnostic criteria have been reported previously. ${ }^{10,18}$

This study was approved by the Institutional Review Board of Peking Union Medical College Hospital. All patients provided written informed consent.

\section{Clinical Treatment and Follow-Up}

All 50 CD patients were treated with TSS, and a final pathological diagnosis was obtained. Clinical data, including age, sex, duration of disease, and years of education, were collected. Duration of disease was defined as the time from first symptom onset until remission after surgery. ${ }^{28}$ Serum cortisol, ACTH, and 24hUFC were measured by direct chemiluminescence immunoassays (Siemens Healthcare Diagnostics Inc.). Remission was defined as serum cortisol decreasing to $<5 \mu \mathrm{g} / \mathrm{dl}$ within 7 days of surgery, in line with the latest Endocrine Society clinical practice guideline. ${ }^{23}$ All CD patients in remission were put on steroid replacement therapy. ${ }^{10}$

At the 3-month follow-up after surgery, MRI was performed with the same scanning parameters as those used preoperatively, and the serum cortisol, ACTH, and 24hUFC levels were measured. Recurrence was defined as an increase in serum cortisol or $24 \mathrm{hUFC}$ above the upper limit of normal in patients who had been in remission. ${ }^{10}$ In all, 46 of the 50 patients $(92.0 \%)$ achieved immediate remission after surgery and had no recurrence at the 3-month follow-up. As for the remaining 4 patients who did not gain immediate remission, 1 patient underwent a second TSS to remove the residual tumor, 1 patient underwent radiotherapy, and at the time of this report 2 patients were undergoing close follow-up, despite negative MRI findings showing no clear tumor residue, because of continued elevated serum cortisol levels at the 3-month follow-up.

\section{MRI Studies}

All participants, including $50 \mathrm{CD}$ patients, both before and after TSS, and $36 \mathrm{HCs}$ underwent brain MRI studies on the same 3-T MR scanner (Discovery MR750; GE) using an 8-channel head-phased array coil. The 3D structural imaging was performed with BRAVO, an optimized 3D fast spoiled gradient-echo sequence $(1 \times 1$ $\mathrm{mm}$ isotropic, preparation time $400 \mathrm{msec}$, flip angle $12^{\circ}$, bandwidth $27.78 \mathrm{~Hz}$ ). All acquisitions were performed by the same technologist. All images were reviewed before postprocessing to exclude those with poor image quality. For intracranial lesions, if a major abnormality such as a hemorrhage, infarct, or cyst was revealed, the subject was excluded from the study.

Image postprocessing was performed with AccuBrain ${ }^{\circledR}$ (BrainNow Medical Technology Limited), an artificial intelligence-assisted web-based brain quantification tool that automatically segments and quantifies brain structures and tissues with good clinical validity. ${ }^{1,41,43}$ Given the MRI data, 3 major brain tissues (white matter, gray matter, and CSF), 12 brain lobes (the left and right frontal, 


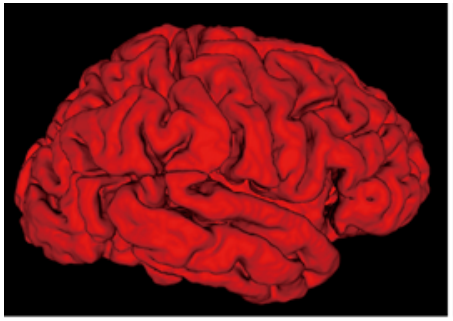

Total Gray Matter

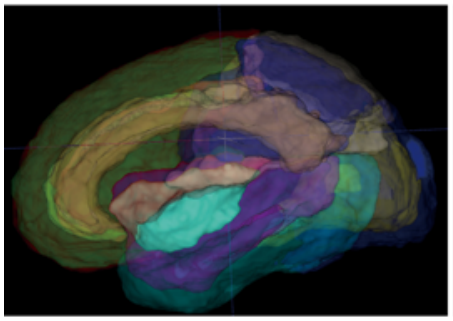

Insula

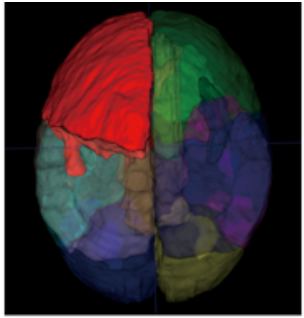

Frontal Lobe

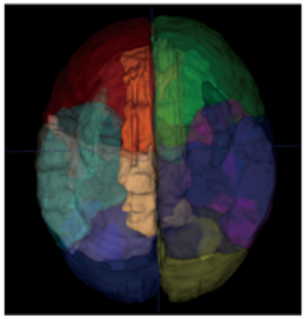

Cingulate Lobe

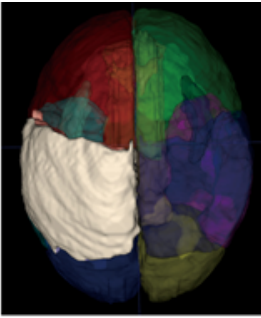

Parietal Lobe

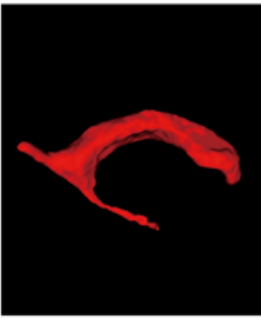

Lateral and Third Ventricle

Occipital Lobe

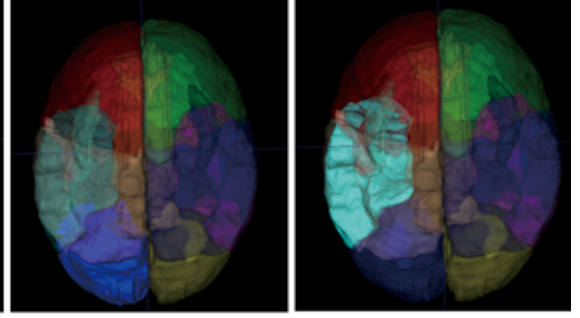

Temporal Lobe

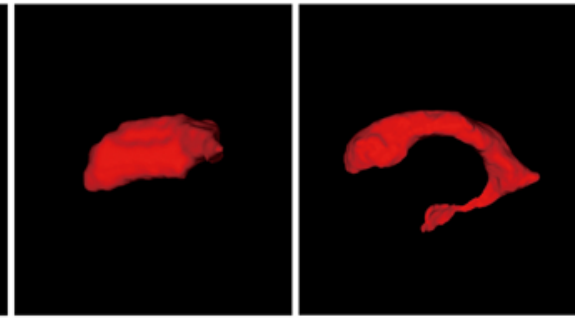

of different brain lobes produced by using Ac-

FIG. 1. Illustrations of $3 D$ reconstructions of brain mapping and autosegmentation of different brain lobes prod
cuBrain ${ }^{\circledast}$ automated brain segmentation and quantification software. Figure is available in color online only. parietal, occipital, and temporal lobes; the left and right insula; and the left and right cingulate lobes) and other brain structures (the hippocampus, amygdala, lateral ventricle, and third ventricle) were segmented and parcellated automatically using the atlas-based segmentation method. The atlas pool, which is embedded in the AccuBrain brain quantification tool, consists of a large number of varied brain templates together with the prior anatomical knowledge specified by experienced radiologists. The anatomical information from the atlas pool was automatically transformed into the individual MRI subject space via an advanced registration process, which serves as the segmentation result of the MRI subject. Based on the segmentation result, the absolute volume (in milliliters) of each brain metric (including brain structure, lobe, and tissue) was calculated and reported as in Fig. 1.

\section{Statistical Analyses}

Data analyses were performed using the statistical package SPSS, version 17 (IBM Corporation). Categorical variables (including sex and cure rate) are presented as a number (percentage). Quantitative data are presented as the mean \pm standard deviation for normally distributed variables (including age, years of education, BMI, and brain volume) or as the median (IQR) for nonnormally distributed variables (including duration of disease and levels of serum cortisol, ACTH, and 24hUFC). Comparisons of categorical variables were performed using chisquare tests. Comparisons of normally distributed numerical variables were performed using independent Student t-tests (including the comparisons between active CD patients and $\mathrm{HCs}$ and between remitted $\mathrm{CD}$ patients and $\mathrm{HCs}$ ). A paired-samples t-test (for normally distributed variables) or Wilcoxon paired rank test (for nonnormally distributed variables) was used for comparisons between active $\mathrm{CD}$ and remitted $\mathrm{CD}$ patients. Here, false discovery rate (FDR) corrections were performed for the group comparisons (independent Student t-tests and paired-sample t-tests) regarding the volumes of multiple brain regions considered in the analyses. Spearman correlations were calculated between brain volume changes and clinical continuous parameters, including age, duration of disease, years of education, and levels of serum cortisol, ACTH, and 24hUFC. Point-biserial correlations were used between brain volume changes and clinical dichotomous variables, such as sex. Since the preoperative brain volumes and hormone levels varied substantially among individual patients, simply using the absolute volume change pre- and postsurgery would not be sufficient. Therefore, the postsurgery volume change percentage relative to the presurgery value was used in the correlation analysis, as described in Starkman et al. ${ }^{35}$ Differences were considered significant at $\mathrm{p}$ values $<0.05$.

\section{Results}

\section{Demographic and Clinical Data}

The active and remitted CD patient and $\mathrm{HC}$ groups did not differ with respect to age, sex, or years of education (Table 1). As expected, levels of serum cortisol, ACTH, and 24hUFC were decreased significantly in remitted CD patients compared with those in preoperative active $\mathrm{CD}$ patients $(\mathrm{p}<0.001)$.

\section{Brain Volume Comparison of the 3 Groups: Active CD, Remitted CD, and $\mathrm{HC}$}

Table 2 shows the volumes of total gray matter, white matter, and brain anatomical structures (including the frontal, parietal, occipital, and temporal lobes, insula, cingulate lobe, hippocampus, amygdala, lateral ventricle, and third ventricle). Comparisons in each region were made between the 3 groups of study participants. Typical MRI 
TABLE 1. Demographic and clinical characteristics of the CD patients and HCs

\begin{tabular}{lcccc}
\hline & Active CD $(\mathrm{n}=50)$ & Remitted CD $(\mathrm{n}=46)$ & HC $(\mathrm{n}=36)$ & $\mathrm{p}$ Value \\
\hline Age, yrs & $31.70 \pm 10.04$ & $32.41 \pm 10.13$ & $35.75 \pm 10.55$ & $0.074 \dagger$ \\
\hline Sex, $\mathrm{n}$ & & & & $0.261 \dagger$ \\
\hline Male & 10 & 10 & 11 & \\
\hline Female & 40 & 36 & $13.25 \pm 3.03$ & $0.481 \dagger$ \\
\hline Education level, yrs & $12.78 \pm 3.05$ & $12.74 \pm 3.14$ & - & - \\
\hline Disease duration, yrs & $3(1,5)$ & $3(1,5)$ & - & $<0.001^{* *} \ddagger$ \\
\hline BMl, $\mathrm{kg} / \mathrm{m}^{2}$ & $26.96 \pm 4.03$ & $25.61 \pm 4.20$ & - & $<0.001^{* *} \ddagger$ \\
\hline Serum cortisol, $\mu \mathrm{g} / \mathrm{dl}$ & $26.67(21.28,32.18)$ & $4.19(1.27,10.66)$ & - & $<0.001^{* *} \ddagger$ \\
\hline ACTH, $\mathrm{pg} / \mathrm{ml}$ & $81.40(49.20,104.00)$ & $19.10(10.33,29.25)$ & - & $<0.001^{* *} \ddagger$ \\
\hline 24hUFC, $\mu \mathrm{gg} / 24 \mathrm{hrs}$ & $565.73(329.79,991.56)$ & $35.47(9.95,113.01)$ & & \\
\hline
\end{tabular}

Values are presented as mean $\pm \mathrm{SD}$ or median (IQR) unless otherwise indicated. ${ }^{* *} p<0.01$.

$\dagger$ Active $\mathrm{CD}$ versus $\mathrm{HC}$.

$\ddagger$ Active versus remitted $C D$.

TABLE 2. Volumetric changes in different brain regions in active and remitted CD patients and HC

\begin{tabular}{|c|c|c|c|c|c|c|}
\hline \multirow[b]{2}{*}{ Brain Region } & \multicolumn{3}{|c|}{ Brain Region Vol (ml) } & \multicolumn{3}{|c|}{$p$ Value } \\
\hline & Active CD Pt & Remitted CD Pt & $\mathrm{HC}$ & Active $\mathrm{CD}$ vs $\mathrm{HC}$ & Active vs Remitted CD & Remitted CD vs HC \\
\hline Gray matter & $669.45 \pm 74.20$ & $694.91 \pm 67.12$ & $724.22 \pm 75.09$ & $0.003^{* *}$ & $0.007^{* *}$ & 0.231 \\
\hline White matter & $454.70 \pm 53.75$ & $457.75 \pm 46.52$ & $459.47 \pm 47.81$ & 0.672 & 0.344 & 0.905 \\
\hline Frontal lobe & $163.14 \pm 18.59$ & $172.38 \pm 18.51$ & $184.81 \pm 22.27$ & $<0.001^{* *}$ & $<0.001^{* *}$ & 0.078 \\
\hline Lt frontal lobe & $81.66 \pm 9.50$ & $86.54 \pm 9.43$ & $93.03 \pm 11.19$ & $<0.001^{* *}$ & $<0.001^{* *}$ & 0.078 \\
\hline Rt frontal lobe & $81.48 \pm 9.30$ & $85.85 \pm 9.29$ & $91.78 \pm 11.21$ & $<0.001^{* *}$ & $<0.001^{* *}$ & 0.078 \\
\hline Parietal lobe & $83.54 \pm 11.06$ & $88.62 \pm 9.26$ & $91.61 \pm 10.18$ & $0.002^{* *}$ & $0.001^{* *}$ & 0.401 \\
\hline Lt parietal lobe & $42.07 \pm 5.58$ & $44.66 \pm 4.86$ & $46.17 \pm 5.12$ & $0.002^{* *}$ & $0.001^{* *}$ & 0.401 \\
\hline Rt parietal lobe & $41.47 \pm 5.65$ & $43.96 \pm 4.60$ & $45.44 \pm 5.39$ & $0.003^{* *}$ & $0.001^{* *}$ & 0.401 \\
\hline Occipital lobe & $74.33 \pm 11.48$ & $77.65 \pm 9.00$ & $79.60 \pm 9.22$ & $0.036^{*}$ & $0.014^{*}$ & 0.524 \\
\hline Lt occipital lobe & $40.57 \pm 6.24$ & $42.08 \pm 5.18$ & $42.93 \pm 4.99$ & 0.087 & $0.024^{*}$ & 0.597 \\
\hline Rt occipital lobe & $33.76 \pm 5.54$ & $35.57 \pm 4.30$ & $36.68 \pm 4.56$ & $0.018^{*}$ & $0.008^{* *}$ & 0.493 \\
\hline Temporal lobe & $122.42 \pm 15.96$ & $127.36 \pm 14.81$ & $135.68 \pm 14.90$ & $0.001^{* *}$ & $0.030^{*}$ & 0.078 \\
\hline Lt temporal lobe & $59.90 \pm 8.33$ & $62.34 \pm 7.50$ & $66.62 \pm 7.39$ & $0.001^{* *}$ & $0.042^{*}$ & 0.078 \\
\hline Rt temporal lobe & $62.52 \pm 7.86$ & $65.02 \pm 7.53$ & $69.06 \pm 7.66$ & $0.001^{* *}$ & $0.022^{*}$ & 0.089 \\
\hline Insula & $14.37 \pm 2.53$ & $15.62 \pm 2.05$ & $16.08 \pm 2.02$ & $0.003^{* *}$ & $0.001^{* *}$ & 0.511 \\
\hline Lt insula & $6.84 \pm 1.21$ & $7.40 \pm 0.97$ & $7.72 \pm 0.90$ & $0.002^{* *}$ & $0.004^{* *}$ & 0.401 \\
\hline Rt insula & $7.52 \pm 1.34$ & $8.22 \pm 1.10$ & $8.37 \pm 1.13$ & $0.006^{* *}$ & $0.001^{* *}$ & 0.645 \\
\hline Cingulate lobe & $29.45 \pm 5.20$ & $31.24 \pm 4.46$ & $32.62 \pm 4.22$ & $0.006^{* *}$ & $0.016^{*}$ & 0.401 \\
\hline Lt cingulate lobe & $14.11 \pm 2.55$ & $14.99 \pm 2.30$ & $15.94 \pm 2.13$ & $0.002^{* *}$ & $0.018^{*}$ & 0.231 \\
\hline Rt cingulate lobe & $15.34 \pm 2.71$ & $16.25 \pm 2.26$ & $16.68 \pm 2.22$ & $0.025^{\star}$ & $0.016^{*}$ & 0.553 \\
\hline Hippocampus & $9.32 \pm 1.15$ & $9.37 \pm 1.15$ & $9.57 \pm 0.83$ & 0.326 & 0.645 & 0.553 \\
\hline Lt hippocampus & $4.55 \pm 0.58$ & $4.58 \pm 0.62$ & $4.66 \pm 0.42$ & 0.372 & 0.546 & 0.597 \\
\hline Rt hippocampus & $4.77 \pm 0.61$ & $4.79 \pm 0.58$ & $4.91 \pm 0.43$ & 0.321 & 0.929 & 0.511 \\
\hline Amygdala & $4.63 \pm 0.67$ & $4.53 \pm 0.52$ & $4.52 \pm 0.43$ & 0.419 & 0.113 & 0.931 \\
\hline Lt amygdala & $2.12 \pm 0.38$ & $2.08 \pm 0.29$ & $2.07 \pm 0.22$ & 0.423 & 0.391 & 0.844 \\
\hline Rt amygdala & $2.51 \pm 0.34$ & $2.45 \pm 0.26$ & $2.46 \pm 0.23$ & 0.423 & 0.059 & 0.905 \\
\hline Lateral ventricle & $18.90 \pm 8.39$ & $16.18 \pm 8.27$ & $13.87 \pm 8.55$ & $0.013^{*}$ & $<0.001^{* *}$ & 0.442 \\
\hline 3rd ventricle & $2.77 \pm 0.90$ & $2.36 \pm 0.84$ & $2.23 \pm 0.84$ & $0.010^{*}$ & $<0.001^{* *}$ & 0.597 \\
\hline
\end{tabular}

$\mathrm{Pt}=$ patient.

Values are presented as mean \pm SD unless otherwise indicated. ${ }^{*} p<0.05$ and ${ }^{* *} p<0.01$. All $p$ values corrected with FDR. 


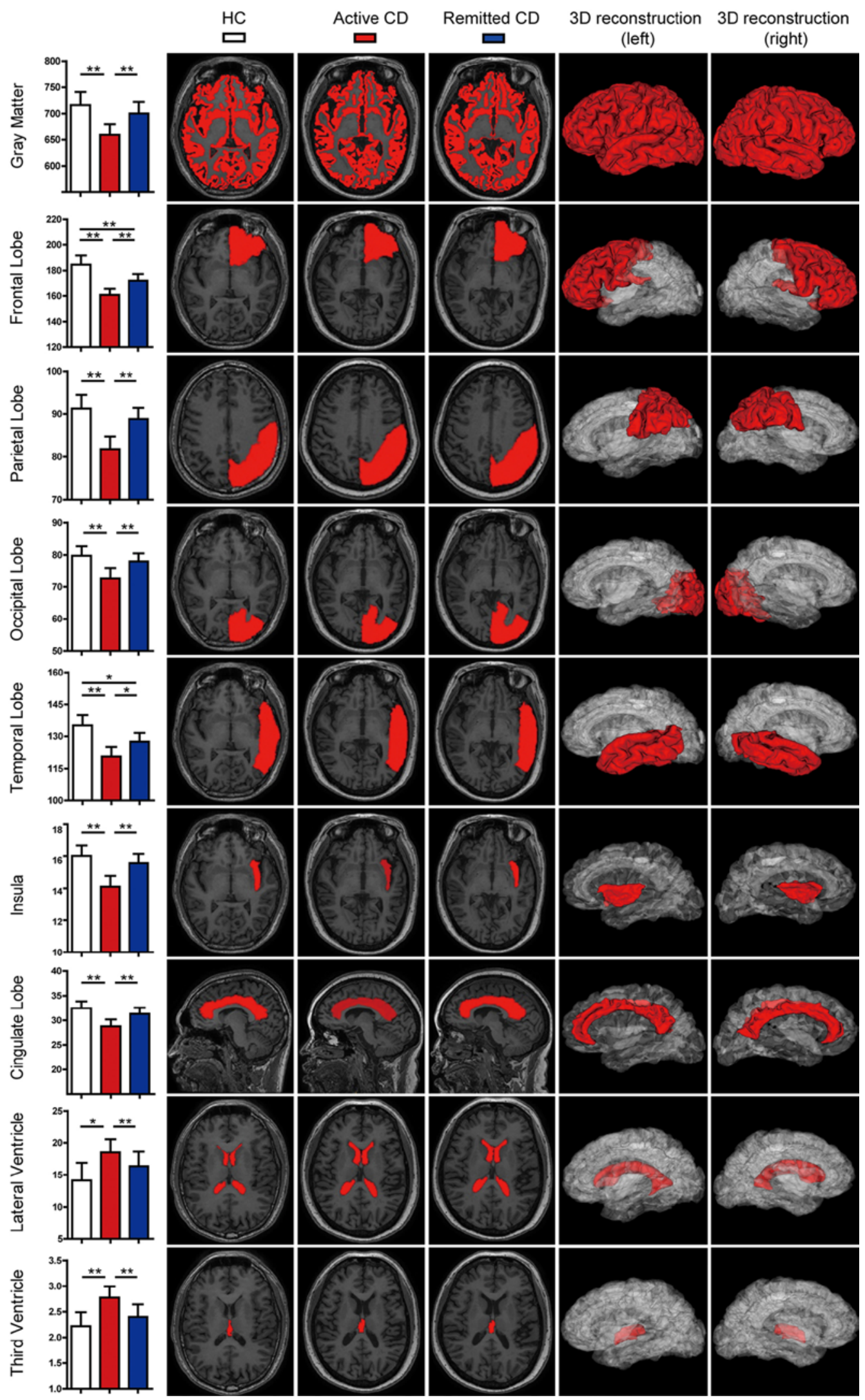

FIG. 2. Bar graphs (far left column) show volumetric comparisons of different brain structures in HCs (white bars) and active CD (red bars) and remitted CD patients (blue bars). MR images (2 left columns and center column) show segmented regions overlaid in red with volumes corresponding to red, white, and blue bars in bar graphs. 3D reconstructed brain images (with left and right views) illustrate segmented regions in 1 patient with active CD. * $p<$ $0.05 ;{ }^{* *} p<0.01$. Figure is available in color online only. 

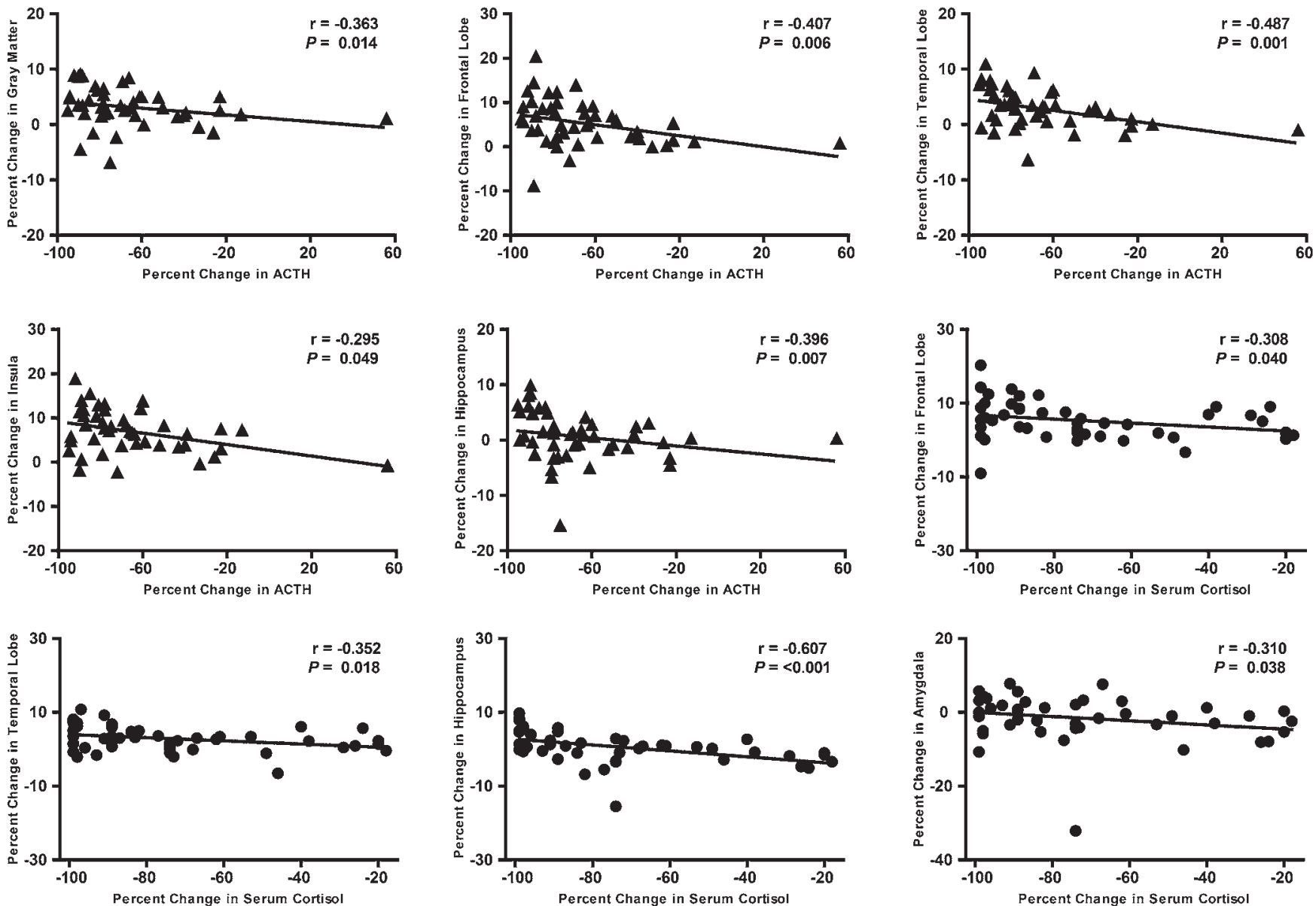

FIG. 3. Correlation analysis of hormone level changes and brain volume changes in different brain regions. Percentage change in ACTH was significantly negatively correlated with changes in the total gray matter $(r=-0.363, p=0.014)$, frontal lobe $(r=-0.407$, $p=0.006)$, temporal lobe $(r=-0.487, p=0.001)$, insula $(r=-0.295, p=0.049)$, and hippocampus $(r=-0.396, p=0.007)$. The percentage change in serum cortisol was significantly negatively correlated with changes in the frontal lobe $(r=-0.308, p=0.04)$, temporal lobe $(r=-0.352, p=0.018)$, hippocampus $(r=-0.607, p<0.001)$, and amygdala $(r=-0.310, p=0.038)$.

image segmentation and 3D reconstruction of brain mapping are shown in Figs. 1 and 2.

\section{Active CD Versus HC}

The total gray matter volume was significantly decreased in the active $\mathrm{CD}$ group compared with that in the HCs $(669.45 \pm 74.20$ vs $724.22 \pm 75.09 \mathrm{ml}, \mathrm{p}<0.001)$, demonstrating whole-brain atrophy in CD patients. Similar significant changes can be seen in most of the brain lobes ( $p<0.05$, corrected with FDR), except for the hippocampus and amygdala ( $p>0.05$, corrected with FDR). The lateral- and third-ventricle volumes in active $\mathrm{CD}$ patients were correspondingly larger than those in HCs $(p<0.05$, corrected with FDR). No significant difference was found in white matter volume between these 2 groups.

\section{Remitted CD Versus Active CD}

At the 3-month follow-up after TSS, $46 \mathrm{CD}$ patients had achieved biological cure. In these patients, brain volumes were compared between the preoperative and postoperative MRI studies. Along with the normalization of serum cortisol, the previously atrophied brain structures, including total gray matter and each brain lobe, all increased significantly in brain volume in remitted CD patients compared with those in active $\mathrm{CD}$ patients $(\mathrm{p}<0.05$, corrected with FDR). Previously abnormally enlarged ventricles (including the lateral ventricle and third ventricle) were all decreased in volume compared with those before surgery ( $\mathrm{p}<0.001$, corrected with FDR).

\section{Remitted CD Versus HC}

Although the results of the above 2 comparisons made at the 3-month follow-up after TSS demonstrated that all previously abnormal brain structures, including brain lobes and ventricles, recovered in the appropriate direction after surgery, we further investigated whether all brain structures had truly recovered to the normal range measured in the HCs. Surprisingly, even though only 3 months had passed since surgery, the overall gray matter volume in remitted CD patients was already not statistically significantly different from that in HCs $(p>0.05$, corrected with FDR). Furthermore, different lobes demonstrated dif- 
ferent recovery speeds. The full-recovery areas, including the parietal lobe, occipital lobe, insula, cingulate lobe, lateral ventricle, and third ventricle, which account for most abnormal brain areas in $\mathrm{CD}$, had already recovered to the normal range $(\mathrm{p}>0.05$, both with and without correction with FDR). The partial recovery areas, including the frontal and temporal lobes, did not fully recover to normal volume within 3 months of surgery ( $p<0.05$, without correction with FDR; and $\mathrm{p}=0.078$, corrected with FDR).

\section{Correlation Analysis}

Spearman and point-biserial correlation analyses revealed no significant correlations between age, duration of disease, or years of education and volume of brain gray matter, white matter, or the third ventricle $(p>0.05)$. Sex was correlated with all brain volumes (gray matter, $\mathrm{p}=$ 0.016 ; white matter, $\mathrm{p}=0.003$; and third ventricle, $\mathrm{p}=$ 0.018 ). However, due to the highly unequal sex distribution in CD patients and in our study (40 females and 10 males), this result should be interpreted with caution.

The correlations of brain structure volume changes with parameter changes were quite different for the 3 common hormones (ACTH, serum cortisol, and 24hUFC) (Fig. 3). The changes in ACTH were significantly and negatively correlated with the change in total gray matter volume (r $=-0.363, p=0.014$ ), which means the recovery of gray matter in the whole brain and the normalization of ACTH were closely related. This correlation was also seen in the frontal lobe $(r=-0.407, p=0.006)$, temporal lobe $(r=$ $-0.487, p=0.001)$, insula $(r=-0.295, p=0.049)$, and hippocampus $(r=-0.396, p=0.007)$. In addition to ACTH, serum cortisol also showed a significant correlation with the frontal lobe $(r=-0.308, p=0.04)$, temporal lobe $(r=$ $-0.352, \mathrm{p}=0.018)$, hippocampus $(\mathrm{r}=-0.607, \mathrm{p}<0.001)$, and amygdala $(\mathrm{r}=-0.310, \mathrm{p}=0.038)$ volumes. Although it did not show a correlation with brain volume changes in total gray matter $(r=-0.191, p=0.208)$, serum cortisol was still considered to be an indispensable biomarker for monitoring the recovery of brain structures in remitted CD patients, as it possesses the strongest correlation in certain areas, such as the hippocampus. Among the 3 hormone parameters, $24 \mathrm{hUFC}$ was the least powerful parameter as it did not correlate with overall gray matter volume or with volumes of each complete lobe separately. Additionally, 24hUFC only correlated significantly with changes in the volume of the left temporal lobe $(r=-0.350, p=0.021)$ and left hippocampus $(r=-0.347, \mathrm{p}=0.023)$.

\section{Discussion}

CD provides a unique model for assessing the effect of cortisol on human brains. ${ }^{5,35}$ The biological cure of CD after TSS offers the opportunity to study whether brain changes caused by excess cortisol are reversible.13 However, this reversibility has long been considered "potential" and remains controversial for several reasons., 3 First, due to the relative rarity of $\mathrm{CD}$, to our knowledge only 19 studies have been conducted, all with rather small sample sizes, leading to low statistical power. ${ }^{3}$ Second, to address the reversibility of brain structure changes in $C D$ patients, preoperative and postoperative brain volumes of the same patients should be compared in a longitudinal cohort. However, in most previous studies (11 of 19), $, 8,16,17,20$, 27-29,32,33,40 which were confined to cross-sectional designs, the active $\mathrm{CD}$ and remitted $\mathrm{CD}$ patients were enrolled from different populations. ${ }^{3}$ Only 8 studies were true longitudinal studies, with the largest cohort including only 27 CD patients. ${ }^{5,12,15,21,35-37,39}$ Third, the controversial results from previous reports concerning the reversibility of brain changes were also partly due to the heterogeneous origin of CS (e.g., pituitary, adrenal, and ectopic), leading to nonidentical clinical features, such as varying durations of hypercortisolism. ${ }^{28}$ Such heterogeneity of the enrolled study population led to even smaller sample sizes in each subgroup. In addition, the existing 8 longitudinal studies all

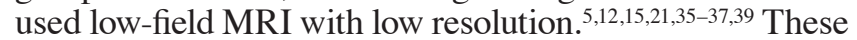
analyses reported manual tracing of brain structures from 2D images or subjective estimation of the degree of cerebral atrophy but not objective or actual 3D volumes. ${ }^{5,35,36}$ Moreover, some MRI data were acquired using routine pituitary protocols instead of whole-brain protocols, leading to incomplete mapping of the entire brain.., 33

Our study, which was performed in the largest longitudinal cohort of $\mathrm{CD}$ patients to date, provides what is to our knowledge the first quantitative confirmation that the volume loss in the gray matter as a whole in CD patients recovers rapidly after surgery, normalizing in most structures within 3 months. Interestingly, the areas most strongly associated with cognition and memory, the frontal and temporal lobes, did not fully recover their volumes by 3 months. This finding deserves further imaging follow-up to determine whether frontal and temporal volumes have a slower rate of full recovery or remain small indefinitely. This first confirmation of brain structural change reversibility in CD will not only help to increase our understanding of this rare disease but also may have important implications for numerous patients who require long-term corticosteroid therapy for autoimmune disorders, cancer, and other disease.

Extensive studies in animal models of the influence of excessive GC on the brain have indicated that effects include impairments of the hippocampus and cingulate lobe. ${ }^{7,30}$ However, human studies are limited, with only 19 studies conducted to date. ${ }^{3}$ The first MRI study was conducted by Starkman et al. ${ }^{33}$ and suggested the atrophy of certain brain regions in $\mathrm{CD}$. However, due to limitations in the technology at that time, the atrophy was measured only by manual tracing of $2 \mathrm{D}$ images or by subjective grading,, 52 and not by using true 3D volumes. In recent years, with the help of modern sophisticated analysis tools, such as FreeSurfer (Laboratory for Computational Neuroimaging at the Athinoula A. Martinos Center for Biomedical Imaging), VBM (University of Jena, Department of Psychiatry), and AccuBrain ${ }^{\circledR}$ (BrainNow Medical Technology Limited), ${ }^{1,13,28,31,41,43}$ some previous conclusions were found to be inaccurate. For example, in early studies performed without knowledge regarding the segmentation of the gray and white matter, ${ }^{13}$ the hippocampus was considered atrophied in CD patients..$^{33,35,36,39}$ However, with modern computer-based autosegmentation and quantification, we found that 3D anatomical volumes of the hippocampus in $\mathrm{CD}$ patients were not significantly different from those in 
the normal HC group in our study. This result is also consistent with studies published in recent years using other automated tools such as FreeSurfer and VBM. ${ }^{13,28}$ Recent reports of widespread reductions in white matter integrity detected using diffusion tensor imaging (DTI) suggest that the hippocampal volume loss seen in earlier studies was likely due to white matter rather than gray matter volume loss. ${ }^{26,40}$

By combining this first use of high-field 3-T MRI in longitudinal studies (the other 8 studies used $1.0 \mathrm{~T}$ or 1.5 $\mathrm{T}^{5,12,15,21,35-37,39}$ ) with autosegmentation and 3D reconstruction techniques, we managed for the first time to perform whole-brain mapping with high anatomical validity in CD patients. Unlike previous studies in which limited brain regions were analyzed using routine pituitary sequences,, 33 our study evaluated the structural changes in $\mathrm{CD}$ at the whole-brain level, comparing different lobes at the same time. The decreased gray matter volume, increased ventricle volume, and unchanged white matter volume observed in this study were all measured by absolute anatomical volume with an accuracy of $0.01 \mathrm{ml}$. Our results demonstrated that, except for the hippocampus and amygdala, most areas in the brain were significantly atrophied, including the frontal lobe, parietal lobe, occipital lobe, temporal lobe, insula, cingulate lobe, lateral ventricle, and third ventricle. Such widespread effects are also consistent with various brain function changes seen in clinical practice, including impairment in cognitive function, mood regulation, and memory. ${ }^{3}$

After TSS in CD patients, along with the return of cortisol levels to the normal range, the patients' psychiatric symptoms were relieved..$^{2,14,25}$ Similar reversibility using anti-GC treatment has been confirmed in animal models of stress. ${ }^{24}$ However, whether the adverse effects of CD are reversible in humans remains controversial ${ }^{3}$ due to the limited number of studies,${ }^{3}$ small sample sizes,${ }^{15}$ various origins of $\mathrm{CS},{ }^{5}$ bias caused by previous radiotherapy, ${ }^{28}$ use of cross-sectional designs, ${ }^{13}$ and other issues. In this present longitudinal cohort, we focused on the dynamic brain structure changes before and after surgery in previously untreated CD patients. After TSS in our patients, the atrophied brain regions in active $\mathrm{CD}$ all changed in an improved direction. The overall gray matter volume in remitted CD patients did not differ from that in HCs only 3 months after surgery. Another novel finding in our study was the nonsynchronized recovery speed of different brain lobes, which was not mentioned in reports of previous studies. By setting the follow-up time point at 3 months after TSS, we distinguished 2 parts in the brain: a full recovery area (including the parietal lobe, occipital lobe, insula, cingulate lobe, lateral ventricle, and third ventricle) and a partial recovery area (including the frontal lobe and temporal lobe). The brain areas responsible for the regulation of memory and cognitive function contain both full recovery areas (such as the cingulate lobe) and partial recovery areas (such as the temporal lobe). This combination of full and partial recovery areas newly found in CD patients could help explain the clinical observations that impaired cognition ${ }^{11,38}$ and memory ${ }^{3,38}$ usually improve after surgery, improvements that are associated with the full-recovery areas we observed. Cognition and memory do not fully recover, however, which according to our findings is due to the combined existence of partial- along with full-recovery areas.

To further investigate the reasons for different brain area recovery speeds, we performed a correlation analysis of brain volume changes and various clinical parameters. Our subregion analysis revealed no correlation of volumes changes with age, duration of disease, or years of education. Furthermore, although the normalizations of hormonal biomarkers (i.e., serum cortisol, ACTH, and 24hUFC) were correlated with brain structure recovery, the strength of the correlation varied among brain regions and biomarkers. Serum cortisol was significantly correlated with recovery in most brain lobes, including the frontal lobe, temporal lobe, hippocampus, and amygdala, mirroring the wide distribution of cortisol receptors in the whole brain, ${ }^{34}$ including mineralocorticoid receptors (MRs) and glucocorticoid receptors (GRs). ${ }^{9}$ The different correlation strengths of serum cortisol within different brain lobes with nonsynchronized recovery speeds are also partially explained by the diversity of MRs and GRs in distribution and downstream regulation. ${ }^{9}$ In addition, corticosteroids control neuronal and network responses in a complex binary fashion by targeting different genes, ${ }^{9}$ which may also explain the difference in correlations between serum cortisol and the various recovery speeds in separate brain regions.

Another novel finding in our study is that ACTH may serve as another useful biomarker, in addition to the wellknown serum cortisol, in the brain recovery process in CD patients. First, ACTH was significantly correlated with total gray matter volume recovery after surgery, whereas serum cortisol and $24 \mathrm{hUFC}$ were not. Additionally, in some brain regions where serum cortisol and ACTH were both correlated with brain volume changes, ACTH levels exhibited stronger correlations. The underlying mechanisms may lie in the dual effects ACTH has on the brain. In addition to the classical steroid-dependent effects through the activation of the hypothalamic-pituitary-adrenocortical (HPA) axis, ACTH can also have direct, steroid-independent effects on the brain through melanocortin receptors, reducing the gene expression of neurons in the brain. ${ }^{6} \mathrm{Nev}-$ ertheless, serum cortisol should never be neglected, since it also has superior correlations with areas such as the hippocampus, which is involved with memory. ${ }^{3}$ Of all 3 CD-related hormones, 24hUFC proved to be the least correlated with brain changes in this longitudinal study. Similar negative results can also be seen in previous cross-sectional studies of the hippocampus ${ }^{33}$ and gray matter thickness. ${ }^{8}$ The recovery of the brain after TSS should be given more attention in clinical practice due to its close relation with various brain functions and health-related quality of life. ${ }^{29,42}$ Serum cortisol, ACTH, and $24 \mathrm{hUFC}$ are all widely used in the postoperative evaluation of CD. However, these variables exhibited different strengths of correlation with brain recovery. Therefore, we recommend a primary reliance on ACTH and serum cortisol as biomarkers of brain recovery when following $\mathrm{CD}$ patients after treatment.

\section{Study Limitations}

There are some limitations of our research. First, al- 
though our study population is to our knowledge the largest longitudinal cohort to date, it included only $50 \mathrm{CD}$ patients, which is far less than the sample size in studies of other common diseases, leading to a relatively low statistical power and potentially more bias. Thus, additional studies including more subjects are needed in the future. Second, questionnaires assessing cognitive impairment and memory deficits may be needed in further analyses to determine if anatomical changes correlate with patient improvement. Third, the initial design of this study was intended primarily to address the lack of previous longitudinal studies investigating short-term reversibility of the brain in remitted CD. Thus, we only set a single followup point at 3 months after TSS. However, we did not anticipate the novel finding of different recovery speeds in separate brain lobes. Further follow-up assessments in the same cohort are needed to determine if the recovery of frontal and temporal lobes in partial recovery areas is merely delayed or incomplete in remitted CD. We are eager to demonstrate dynamic brain volumetric changes in $\mathrm{CD}$ in the long term. Last but not least, this structural MRI study failed to distinguish whether the increases in the volumes of brain structures after TSS were the result of more brain cells or simply due to the brain cells retaining higher volumes of fluid. Further functional MRI or DTI studies, as well as animal and cytological experiments, are still needed to discover the underlying mechanisms of brain recovery found in this study.

\section{Conclusions}

Our findings provide what is to our knowledge the first demonstration of the rapid reversal of total gray matter volume loss in short-term remitted CD. Equally importantly, we demonstrated the combination of full recovery areas and partial recovery areas after TSS, consistent with the incomplete recovery of memory and cognitive function observed in clinical practice. ACTH and serum cortisol are suggested to be reliable serum biomarkers of brain recovery after surgery for clinical use. Finally, multimodal imaging (functional MRI, DTI) studies and functional assessments are needed to elucidate the mechanisms underlying the differential recovery speeds of brain areas in the future.

\section{Acknowledgments}

This study was funded by the China Postdoctoral Science Foundation (2019M650567) and the Clinical Application Research of the Capital Characteristic Fund from the Beijing Municipal Science and Technology Commission (Z151100004015099).

\section{References}

1. Abrigo J, Shi L, Luo Y, Chen Q, Chu WCW, Mok VCT: Standardization of hippocampus volumetry using automated brain structure volumetry tool for an initial Alzheimer's disease imaging biomarker. Acta Radiol 60:769-776, 2019

2. Andela CD, van der Werff SJ, Pannekoek JN, van den Berg SM, Meijer OC, van Buchem MA, et al: Smaller grey matter volumes in the anterior cingulate cortex and greater cerebellar volumes in patients with long-term remission of Cushing's disease: a case-control study. Eur J Endocrinol 169:811819,2013
3. Andela CD, van Haalen FM, Ragnarsson O, Papakokkinou E, Johannsson G, Santos A, et al: Mechanisms in endocrinology: Cushing's syndrome causes irreversible effects on the human brain: a systematic review of structural and functional magnetic resonance imaging studies. Eur J Endocrinol 173:R1-R14, 2015

4. Biller BM, Grossman AB, Stewart PM, Melmed S, Bertagna $\mathrm{X}$, Bertherat J, et al: Treatment of adrenocorticotropin-dependent Cushing's syndrome: a consensus statement. J Clin Endocrinol Metab 93:2454-2462, 2008

5. Bourdeau I, Bard C, Noël B, Leclerc I, Cordeau MP, Bélair $\mathrm{M}$, et al: Loss of brain volume in endogenous Cushing's syndrome and its reversibility after correction of hypercortisolism. J Clin Endocrinol Metab 87:1949-1954, 2002

6. Brunson KL, Khan N, Eghbal-Ahmadi M, Baram TZ: Corticotropin (ACTH) acts directly on amygdala neurons to downregulate corticotropin-releasing hormone gene expression. Ann Neurol 49:304-312, 2001

7. Cerqueira JJ, Catania C, Sotiropoulos I, Schubert M, Kalisch $\mathrm{R}$, Almeida OF, et al: Corticosteroid status influences the volume of the rat cingulate cortex - a magnetic resonance imaging study. J Psychiatr Res 39:451-460, 2005

8. Crespo I, Esther GM, Santos A, Valassi E, Yolanda VG, De Juan-Delago M, et al: Impaired decision-making and selective cortical frontal thinning in Cushing's syndrome. Clin Endocrinol (Oxf) 81:826-833, 2014

9. de Kloet ER, Joëls M, Holsboer F: Stress and the brain: from adaptation to disease. Nat Rev Neurosci 6:463-475, 2005

10. Feng M, Liu Z, Liu X, Bao X, Yao Y, Deng K, et al: Diagnosis and outcomes of 341 patients with Cushing's disease following transsphenoid surgery: a single-center experience. World Neurosurg 109:e75-e80, 2018

11. Forget H, Lacroix A, Cohen H: Persistent cognitive impairment following surgical treatment of Cushing's syndrome. Psychoneuroendocrinology 27:367-383, 2002

12. Hook JN, Giordani B, Schteingart DE, Guire K, Giles J, Ryan $\mathrm{K}$, et al: Patterns of cognitive change over time and relationship to age following successful treatment of Cushing's disease. J Int Neuropsychol Soc 13:21-29, 2007

13. Jiang H, Ren J, He NY, Liu C, Sun YH, Jian FF, et al: Volumetric magnetic resonance imaging analysis in patients with short-term remission of Cushing's disease. Clin Endocrinol (Oxf) 87:367-374, 2017

14. Kelly WF: Psychiatric aspects of Cushing's syndrome. QJM 89:543-551, 1996

15. Khiat A, Bard C, Lacroix A, Boulanger Y: Recovery of the brain choline level in treated Cushing's patients as monitored by proton magnetic resonance spectroscopy. Brain Res 862:301-307, 2000

16. Khiat A, Bard C, Lacroix A, Rousseau J, Boulanger Y: Brain metabolic alterations in Cushing's syndrome as monitored by proton magnetic resonance spectroscopy. NMR Biomed 12:357-363, 1999

17. Langenecker SA, Weisenbach SL, Giordani B, Briceño EM, Guidotti Breting LM, Schallmo MP, et al: Impact of chronic hypercortisolemia on affective processing. Neuropharmacology 62:217-225, 2012

18. Liu Y, Liu X, Hong X, Liu P, Bao X, Yao Y, et al: Prediction of recurrence after transsphenoidal surgery for Cushing's disease: the use of machine learning algorithms. Neuroendocrinology 108:201-210, 2019

19. Lonser RR, Nieman L, Oldfield EH: Cushing's disease: pathobiology, diagnosis, and management. J Neurosurg 126:404-417, 2017

20. Maheu FS, Mazzone L, Merke DP, Keil MF, Stratakis CA, Pine DS, et al: Altered amygdala and hippocampus function in adolescents with hypercortisolemia: a functional magnetic resonance imaging study of Cushing syndrome. Dev Psychopathol 20:1177-1189, 2008 
21. Merke DP, Giedd JN, Keil MF, Mehlinger SL, Wiggs EA, Holzer S, et al: Children experience cognitive decline despite reversal of brain atrophy one year after resolution of Cushing syndrome. J Clin Endocrinol Metab 90:2531-2536, 2005

22. Newell-Price J, Bertagna X, Grossman AB, Nieman LK: Cushing's syndrome. Lancet 367:1605-1617, 2006

23. Nieman LK, Biller BM, Findling JW, Murad MH, NewellPrice J, Savage MO, et al: Treatment of Cushing's syndrome: an Endocrine Society clinical practice guideline. J Clin Endocrinol Metab 100:2807-2831, 2015

24. Oomen CA, Mayer JL, de Kloet ER, Joëls M, Lucassen PJ: Brief treatment with the glucocorticoid receptor antagonist mifepristone normalizes the reduction in neurogenesis after chronic stress. Eur J Neurosci 26:3395-3401, 2007

25. Pereira AM, Tiemensma J, Romijn JA: Neuropsychiatric disorders in Cushing's syndrome. Neuroendocrinology 92 (Suppl 1):65-70, 2010

26. Pires P, Santos A, Vives-Gilabert Y, Webb SM, Sainz-Ruiz A, Resmini E, et al: White matter alterations in the brains of patients with active, remitted, and cured Cushing syndrome: a DTI study. AJNR Am J Neuroradiol 36:1043-1048, 2015

27. Resmini E, Santos A, Gómez-Anson B, López-Mourelo O, Pires P, Vives-Gilabert Y, et al: Hippocampal dysfunction in cured Cushing's syndrome patients, detected by ${ }^{1} \mathrm{H}-\mathrm{MR}$ spectroscopy. Clin Endocrinol (Oxf) 79:700-707, 2013

28. Resmini E, Santos A, Gómez-Anson B, Vives Y, Pires P, Crespo I, et al: Verbal and visual memory performance and hippocampal volumes, measured by 3-Tesla magnetic resonance imaging, in patients with Cushing's syndrome. J Clin Endocrinol Metab 97:663-671, 2012

29. Santos A, Resmini E, Crespo I, Pires P, Vives-Gilabert Y, Granell E, et al: Small cerebellar cortex volume in patients with active Cushing's syndrome. Eur J Endocrinol 171:461469, 2014

30. Sapolsky RM, Uno H, Rebert CS, Finch CE: Hippocampal damage associated with prolonged glucocorticoid exposure in primates. J Neurosci 10:2897-2902, 1990

31. Shi L, Wang D, Liu S, Pu Y, Wang Y, Chu WC, et al: Automated quantification of white matter lesion in magnetic resonance imaging of patients with acute infarction. J Neurosci Methods 213:138-146, 2013

32. Simmons NE, Do HM, Lipper MH, Laws ER Jr: Cerebral atrophy in Cushing's disease. Surg Neurol 53:72-76, 2000

33. Starkman MN, Gebarski SS, Berent S, Schteingart DE: Hippocampal formation volume, memory dysfunction, and cortisol levels in patients with Cushing's syndrome. Biol Psychiatry 32:756-765, 1992

34. Starkman MN, Giordani B, Berent S, Schork MA, Schteingart DE: Elevated cortisol levels in Cushing's disease are associated with cognitive decrements. Psychosom Med 63:985-993, 2001

35. Starkman MN, Giordani B, Gebarski SS, Berent S, Schork MA, Schteingart DE: Decrease in cortisol reverses human hippocampal atrophy following treatment of Cushing's disease. Biol Psychiatry 46:1595-1602, 1999

36. Starkman MN, Giordani B, Gebarski SS, Schteingart DE: Improvement in learning associated with increase in hippocampal formation volume. Biol Psychiatry 53:233-238, 2003
37. Starkman MN, Giordani B, Gebarski SS, Schteingart DE: Improvement in mood and ideation associated with increase in right caudate volume. J Affect Disord 101:139-147, 2007

38. Tiemensma J, Kokshoorn NE, Biermasz NR, Keijser BJ, Wassenaar MJ, Middelkoop HA, et al: Subtle cognitive impairments in patients with long-term cure of Cushing's disease. J Clin Endocrinol Metab 95:2699-2714, 2010

39. Toffanin T, Nifosì F, Follador H, Passamani A, Zonta F, Ferri $\mathrm{G}$, et al: Volumetric MRI analysis of hippocampal subregions in Cushing's disease: a model for glucocorticoid neural modulation. Eur Psychiatry 26:64-67, 2011

40. van der Werff SJ, Andela CD, Nienke Pannekoek J, Meijer OC, van Buchem MA, Rombouts SA, et al: Widespread reductions of white matter integrity in patients with long-term remission of Cushing's disease. Neuroimage Clin 4:659667,2014

41. Wang C, Zhao L, Luo Y, Liu J, Miao P, Wei S, et al: Structural covariance in subcortical stroke patients measured by automated MRI-based volumetry. Neuroimage Clin 22:101682, 2019

42. Webb SM, Badia X, Barahona MJ, Colao A, Strasburger CJ, Tabarin A, et al: Evaluation of health-related quality of life in patients with Cushing's syndrome with a new questionnaire. Eur J Endocrinol 158:623-630, 2008

43. Zhao W, Luo Y, Zhao L, Mok V, Su L, Yin C, et al: Automated brain MRI volumetry differentiates early stages of Alzheimer's disease from normal aging. J Geriatr Psychiatry Neurol 32:354-364, 2019

\section{Disclosures}

Dr. Shi reports ownership in BrainNow, Ltd. Dr. Luo is an employee of BrainNow, Ltd.

\section{Author Contributions}

Conception and design: Xing, Hou, Gao, Shi, Zhu, Lu, R Wang, F Feng. Acquisition of data: Hou, Gao, Guo, Zhu, Lu, Z Wang, M Feng, Bao, R Wang, F Feng. Analysis and interpretation of data: Xing, Hou, Gao, Shi, Luo, Young, Qin. Drafting the article: Hou, Gao. Critically revising the article: Xing, Hou, Gao, Shi, Young, Qin. Reviewed submitted version of manuscript: all authors. Approved the final version of the manuscript on behalf of all authors: Xing. Statistical analysis: Hou, Gao, Luo, Bao. Administrative/technical/material support: Xing, Hou, Gao, Shi, Luo, Young, Qin, M Feng, Bao, F Feng. Study supervision: Xing, Hou, Gao, F Feng.

\section{Supplemental Information}

\section{Previous Presentations}

Portions of this work were presented as a Hot Topic oral presentation at the Pituitary Society 16th International Pituitary Congress, New Orleans, Louisiana, March 21, 2019.

\section{Correspondence}

Bing Xing: Peking Union Medical College Hospital, Beijing, China.xingbingemail@aliyun.com. 